\title{
Potential Pediatric Applications of PET/MR
}

\author{
Sandra Purz ${ }^{* 1}$, Osama Sabri*1, Adrian Viehweger ${ }^{2}$, Henryk Barthel $^{1}$, Regine Kluge ${ }^{1}$, Ina Sorge ${ }^{2}$, \\ and Franz Wolfgang Hirsch ${ }^{2}$ \\ ${ }^{I}$ Department of Nuclear Medicine, University Hospital of Leipzig, Leipzig, Germany; and ${ }^{2}$ Department of Pediatric Radiology, \\ University Hospital of Leipzig, Leipzig, Germany
}

\begin{abstract}
Medical imaging with multimodality and whole-body technologies has continuously improved in recent years. The advent of combined modalities such as PET/CT and PET/MR offers new tools with an exact fusion of molecular imaging and high-resolution anatomic imaging. For noninvasive pediatric diagnostics, molecular imaging and whole-body MR have become important, especially in pediatric oncology. Because it has a lower radiation exposure than PET/CT, combined PET/MR is expected to be of special use in pediatric diagnostics. This review focuses on possible pediatric applications of PET/MR hybrid imaging, particularly pediatric oncology and neurology but also the diagnosis of infectious or inflammatory diseases.
\end{abstract}

Key Words: pediatrics; pediatric oncology; oncology; PET/MR

J Nucl Med 2014; 55:32S-39S

DOI: 10.2967/jnumed.113.129304

I maging plays a key role in the detection, staging, and monitoring of diseases. Nuclear medicine imaging methods such as PET allow the localization and visualization of functional processes with small amounts of radiopharmaceuticals, whereas radiologic imaging methods such as ultrasound, CT, and MR provide detailed anatomic information.

The aim of combining functional PET imaging with MR imaging, yielding high soft-tissue contrast, is to improve image fusion and anatomic resolution. However, hybrid imaging is also expected to lead to deeper insights into the dynamics and characteristics of tumoral or inflammatory processes (1-3). Thus, PET/MR will provide new perspectives for noninvasive imaging in clinical management and research (4). Several studies have already proved the feasibility of PET/MR in a clinical setting, with high image quality in an acceptable examination time and no loss of information, compared with the results of conventional PET/CT (4-6).

Because ALARA (as low as reasonably achievable) principles should be considered for all imaging studies, one of the most important reasons for establishing PET/MR in pediatric patients is that PET/MR has a lower radiation burden than PET/CT $(1,7,8)$. This fact may be especially significant for pediatric cancer

Received Nov. 7, 2013; revision accepted Mar. 7, 2014

For correspondence or reprints contact: Sandra Purz, Department of Nuclear Medicine, University of Leipzig, Liebigstrasse 18, 04103 Leipzig, Germany.

E-mail: sandra.purz@medizin.uni-leipzig.de

${ }^{*}$ Contributed equally to this work.

Published online Apr. 24, 2014.

COPYRIGHT (c) 2014 by the Society of Nuclear Medicine and Molecular Imaging, Inc. patients, who may need to undergo repeated diagnostic imaging sessions. An advantage of whole-body imaging-and therefore also of combined PET/MR - in pediatric patients is the reduction of multiple regional examinations, which may result in a more rapid initiation of treatment. In addition, repeated sedation or anesthesia of pediatric patients may be required less frequently. Disadvantages of whole-body MR are the limited spatial resolution of the images, compared with that of a dedicated local investigation, and the smaller number of diagnostic sequences (9).

The imaging protocols for PET/MR, like those for combined PET/CT, should be kept as simple as possible to maintain patient tolerance (10). In general, MR image contrast may be weighted (e.g., T1 and T2) to demonstrate different anatomic structures or pathologies. Faster imaging techniques (e.g., parallel image acquisition methods) and table movement techniques can allow faster whole-body examinations (45-60 min), an advantage of particular importance for young patients. Functional MR (fMR) is a more recent development combining anatomic information and biophysiologic information; it includes diffusion-weighted imaging (DWI), which reflects cell density, or dynamic contrast-enhanced imaging, which depicts blood vessels generated by a tumor. The incorporation of fMR into PET/MR protocols may result in longer scanning times, which may be less well tolerated, especially by pediatric patients. Thus, the complementary benefit of whole-body MR coupled with advanced MR sequences, such as DWI, needs to be evaluated $(11,12)$.

For pediatric patients, data on the clinical potential of PET/MR are rare. A recommendation for a child-specific sequence protocol for PET/MR imaging, involving a combination of whole-body imaging to determine the spread of cancer and additional dedicated local sequences to provide a more detailed visualization in the region of the primary tumor, was made by Hirsch et al. (8). On the basis of our experience with pediatric whole-body MR, a water-sensitive fast inversion recovery sequence is used for whole-body imaging, and T1-weighted gradient-echo sequences are used in the abdominal region because of a preferred shorter acquisition time. All sequences in the thoracic region and upper abdomen are performed with respiratory triggering. For that purpose, a respiratory belt has been found to be more stable than navigator sequences in children. Artifacts due to movements of the diaphragm are eliminated very effectively by this technique.

This review highlights potential clinical applications of PET/ MR in pediatric oncology, neurology, and inflammation.

\section{POTENTIAL APPLICATIONS IN PEDIATRIC ONCOLOGY}

Imaging techniques such as ultrasound, MR, CT, PET, and SPECT have been implemented in oncologic workup with the understanding that each modality has advantages and disadvantages. The aim of contemporary medical imaging is to integrate 
complementary imaging to increase information earned by a single modality (13). Tumor imaging not only is used for tumor detection and evaluation of tumor characteristics and tumor extension but also has a role in the assessment of a treatment response and surveillance for residual or recurrent tumor $(10,14)$. Recently, Buchbender et al. $(15,16)$ presented a review about the use of PET/MR in oncology. Drzezga et al. (5) demonstrated that the reliability of PET/MR for the detection of hypermetabolic tumor lesions in oncology patients was comparable to that of PET/CT.

\section{Lymphoma}

Non-Hodgkin lymphoma and Hodgkin lymphoma account for about $10 \%$ and $18 \%$ of all childhood cancers, respectively; both have high cure rates $(10,17,18)$.

For both diseases, imaging plays an important role, especially for accurate determination of the extent of disease; the latter is an essential part of lymphoma staging and has an impact on treatment decisions (15). The aim is to achieve a favorable outcome and, particularly in pediatric patients, to avoid unnecessary side effects and long-term consequences of therapy. Conventional imaging techniques such as CT, MR, and ultrasound are frequently used for lymphoma in pediatric patients; however, their main drawback is that the assessment of nodal involvement is based on size criteria, which may be inaccurate $(19,20)$.

The use of ${ }^{18} \mathrm{~F}-\mathrm{FDG}$ PET for lymphoma has been assessed extensively in numerous studies $(17,19) .{ }^{18} \mathrm{~F}-\mathrm{FDG}$ PET and $\mathrm{PET} / \mathrm{CT}$ are now recognized as valuable staging procedures for most lymphoma subtypes, with a high sensitivity in patients with classic Hodgkin lymphoma and aggressive non-Hodgkin lymphoma (19). In a retrospective analysis, Montravers et al. (17) found that ${ }^{18} \mathrm{~F}$-FDG PET was sensitive for staging and restaging of disease, showing more lesions than conventional imaging. The authors also reported high accuracies for monitoring the response to therapy and for characterizing residual masses, which occur frequently in approximately two thirds of patients with Hodgkin lymphoma and which mostly represent fibrosis rather than active disease. A more recent comparison of ${ }^{18} \mathrm{~F}-\mathrm{FDG}$ PET/CT and conventional imaging for a regional whole-body analysis revealed sensitivities and specificities of $95.9 \%$ and $99.7 \%$, respectively, for ${ }^{18} \mathrm{~F}-\mathrm{FDG} \mathrm{PET} / \mathrm{CT}$ and $70.1 \%$ and $99.0 \%$, respectively, for conventional imaging (21).

Whole-body MR, with a high sensitivity (96\%), allows a wholebody overview, which is particularly useful in diagnostic procedures for malignant disease $(19,22)$. The clinical application of wholebody fMR with DWI is currently under investigation, and this technique is also being compared with ${ }^{18} \mathrm{~F}-\mathrm{FDG}$ PET/CT (23). Gu et al. (19) evaluated conventional whole-body MR without and with DWI in the detection of known ${ }^{18} \mathrm{~F}-\mathrm{FDG}$-avid lymphomas in 17 adult patients. An additive value of DWI over T2-weighted sequences alone was reported, improving the accuracy of detecting nodal disease in the abdomen and pelvis and extranodal disease without changing the overall disease stage. Lin et al. (23) hypothesized that because of the excellent soft-tissue contrast, whole-body DWI may help to identify additional lesions, in particular, extranodal involvement in the liver, spleen, and kidneys, relative to the findings obtained with ${ }^{18}$ F-FDG PET/CT. In a more recent study with 39 children and adolescents, Punwani et al. (11) demonstrated the use of DWI to be highly complementary to ${ }^{18} \mathrm{~F}$-FDG PET, supporting its incorporation into integrated PET/MR protocols for lymphoma.

The evaluation of bone marrow infiltration is an essential step in the staging of lymphoma because it defines stage IV of Hodgkin lymphoma according to the Ann Arbor classification. ${ }^{18}$ F-FDG PET has been reported to have higher sensitivity and specificity than biopsy of bone marrow taken from the iliac crest in detecting bone marrow involvement in pediatric Hodgkin lymphoma, which typically shows a multifocal pattern in the central skeleton (24). A meta-analysis by Wu et al. (25) demonstrated almost equal sensitivities ( $\sim 90 \%$ ) of ${ }^{18} \mathrm{~F}$-FDG PET/CT and MR for diagnosing lymphoma with bone marrow involvement. The specificity of MR has been reported to be lower than that of ${ }^{18} \mathrm{~F}$-FDG PET because posttherapeutic changes, such as bone marrow edema, necrotic tissue, or age-dependent changes in the appearance of bone marrow in children, may lead to misinterpretation $(25,26)$. A retrospective analysis of the diagnostic value of combined and coregistered ${ }^{18}$ F-FDG PET/MR in pediatric oncology (mainly lymphoma) did not miss any bone or bone marrow lesions relative to an analysis of each modality separately. Although CT has been described as the gold standard for the detection of lung metastases, in some radiology centers lung MR has become an established alternative for the assessment of pulmonary diseases, especially for patients in whom radiation exposure should be avoided. For the detection of lung nodules, MR has been described as superior to radiography and almost as sensitive as CT (27). With the use of an adequate examination technique including triggered T2weighted turbo-spin-echo sequences, pulmonary metastases with a 5-mm diameter could be diagnosed (28).

For restaging and early response assessment, numerous studies have demonstrated the value of ${ }^{18} \mathrm{~F}-\mathrm{FDG}$ PET. The main advantage of PET in these settings is its ability to distinguish between viable tumor and fibrosis in residual masses. Conventional imaging methods such as CT and MR generally struggle in making this distinction (29-31). In children with Hodgkin lymphoma, data on early response assessment with ${ }^{18}$ F-FDG PET have demonstrated a high negative predictive value, with an excellent prognosis for children showing a normalization of ${ }^{18}$ F-FDG PET findings at an early time during chemotherapy $(30,31)$. This early prediction of the response to therapy allows risk-adapted tumor treatment with less intensive and less toxic regimens. Thus, the risk of secondary malignancies due to late effects of intensive chemotherapy and radiotherapy could be reduced, especially beneficial for pediatric patients (32). There is evidence that fMR, especially with DWI, may have a role in restaging and therapy response assessment (15). Chen et al. (33) observed significant increases in apparent diffusion coefficient values in adult patients with non-Hodgkin lymphoma after only a few days of chemotherapy, far earlier than morphologic changes.

Thus, the use of combined PET/fMR instead of PET/CT in the management of lymphoma is expected to be advantageous for staging (Fig. 1), restaging, and therapy response evaluation. Furthermore, it may facilitate differentiation from recurrent lymphoma and thymic rebound, which is frequently seen in children undergoing chemotherapy (15).

Regarding the added value of fMR sequences in the diagnostic management of malignant lymphoma and their implementation into PET/MR protocols, comparative studies with conventional imaging techniques are essential before definitive conclusions can be drawn. This requirement is especially important for DWI because the visualization of both nonmalignant and malignant lymph nodes has been described as an important limitation of this technique (34).

\section{Primary Bone Tumors}

Osteosarcoma and Ewing sarcoma are the most frequently occurring primary bone malignancies of childhood and adolescence. 


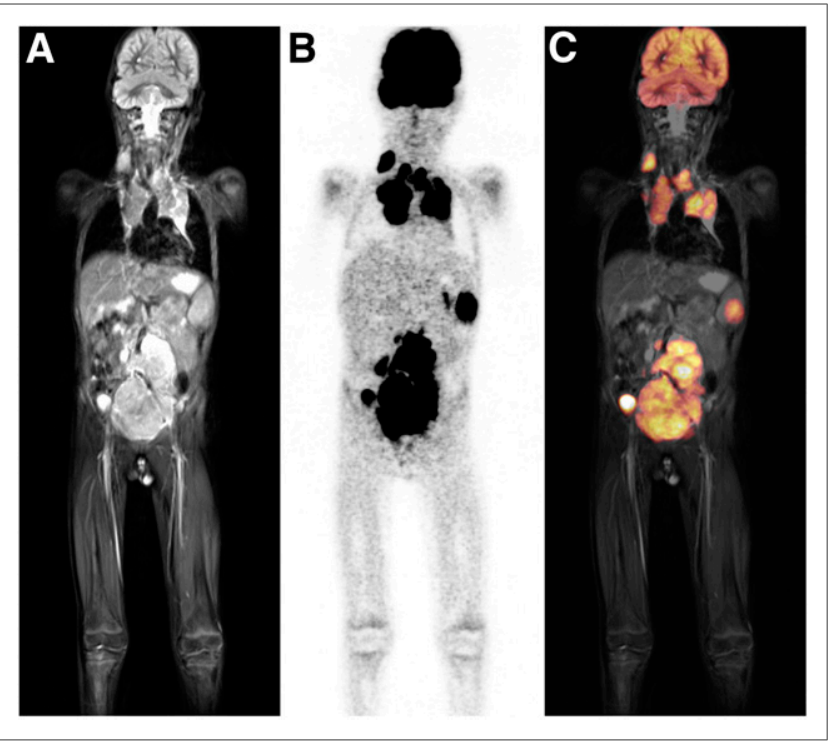

FIGURE 1. Six-year-old boy with newly diagnosed Hodgkin lymphoma. Pretherapeutic T2-weighted whole-body MR imaging (A) and simultaneous ${ }^{18} \mathrm{~F}-\mathrm{FDG}$ PET/MR imaging ( $\mathrm{B}$ and $\mathrm{C}$ ) show multiple hypermetabolic tumor lesions on both sides of diaphragm, in accordance with stage IV in Ann Arbor classification.

Both malignancies are typically located in the extremities but, especially for Ewing sarcoma, pelvic, rib, and vertebral lesions can also occur $(10,35)$. Beyond conventional radiography, MR is the preferred modality for diagnosis and local tumor staging, whereas nodal staging may benefit from ${ }^{18}$ F-FDG PET (15). According to the European Sarcoma Network Working Group (35), extensive staging should be performed to assess the extent of distant disease and should include bone scintigraphy, chest radiography, and chest CT for the evaluation of lung involvement.

Although the value of functional imaging for the staging and follow-up of malignant bone tumors has not yet been established, some promising results can be found in the literature (15). Denecke et al. (36) evaluated ${ }^{18} \mathrm{~F}$-FDG PET and MR as tools for noninvasive therapy response assessment in 27 pediatric patients with bone sarcoma (Ewing sarcoma in 16/27 and osteosarcoma in $11 / 27$ ) before local tumor resection. For the subgroup of patients with osteosarcoma, ${ }^{18}$ F-FDG PET significantly discriminated responders from nonresponders when a reduction in the standardized uptake value and the absolute posttherapeutic standardized uptake value were used. Interestingly, for the subgroup of patients with Ewing sarcoma, ${ }^{18}$ F-FDG PET was not beneficial for discrimination. The volume reduction measured by MR or CT did not significantly discriminate responders from nonresponders in either subgroup. In contrast, Hawkins et al. (37) investigated the value of the therapy response demonstrated by ${ }^{18} \mathrm{~F}$-FDG PET for predicting outcome in patients with Ewing sarcoma. Their results demonstrated that a standardized uptake value of less than 2.5 after neoadjuvant chemotherapy was associated with an improvement in progression-free survival. In a retrospective analysis, London et al. (38) compared PET/CT and conventional imaging for pediatric primary bone tumors. The authors reported that PET/CT appeared to be more accurate than conventional imaging for detecting metastases from pediatric primary bone tumors, excluding lung lesions. Compared with a sensitivity of $83 \%$ and a specificity of $78 \%$ for conventional imaging, the $98 \%$ sensitivity and
97\% specificity of PET/CT suggest that the latter is more accurate. fMR, especially with DWI or dynamic contrast enhancement, has been described as permitting the recognition of tumor necrosis induced by chemotherapy in osteosarcomas and therefore may also be used for therapy response assessment (39).

In summary, both ${ }^{18} \mathrm{~F}$-FDG PET and MR are of value for staging, restaging, and therapy response assessment for primary bone tumors. Although combined PET/MR is not expected to increase the diagnostic accuracy of tumor staging over MR alone, the staging of nodal and distant metastases is expected to benefit from the combination. Furthermore, the PET component may help to guide diagnostic biopsies, maximizing the accuracies of staging and grading and influencing treatment decisions and outcome (15). For the detection of pulmonary metastases from primary bone tumors, a clear superiority of chest CT over ${ }^{18}$ F-FDG PET has been shown (40).

\section{Soft-Tissue Tumors}

Pediatric soft-tissue tumors may be present as rhabdomyosarcoma, nonrhabdomyosarcoma soft-tissue sarcomas compromising various mesenchymal malignancies, or desmoid tumors (41). The staging of soft-tissue tumors requires an assessment of primary tumor size, location, and invasiveness. Because of its high softtissue contrast, the main imaging modality for the diagnosis and staging of soft-tissue sarcomas typically is MR, especially in children $(15,42)$. Additional PET data will not obviate a histopathologic diagnosis by biopsy; therefore, combined PET/MR is not expected to be of special use for primary local tumor diagnosis (15). For staging purposes, the results of a prospective multicenter trial of 46 pediatric and adolescent patients indicated a marked added value of ${ }^{18}$ F-FDG PET over conventional imaging for the detection of additional lesions and a relevant impact on therapy planning (40). Ricard et al. (43) evaluated the additional benefit of ${ }^{18}$ F-FDG PET/CT for staging and follow-up in 13 pediatric patients with rhabdomyosarcoma; they concluded that ${ }^{18} \mathrm{~F}-\mathrm{FDG}$ PET/CT was useful, especially for assessing lymph node and bone involvement and for cases in which the primary sites of rhabdomyosarcoma were unclear. By analogy, whole-body PET/MR may have great potential to become an integrated part of the diagnostic algorithm in the future (15).

Quantitative ${ }^{18}$ F-FDG PET has been described to be significantly more accurate than size-based criteria for assessing the histopathologic response to neoadjuvant therapy of high-grade soft-tissue sarcomas, and its consideration as the modality of choice for monitoring the treatment response has been recommended (44). This recommendation is in accord with a meta-analysis by Piperkova et al. (45), which showed ${ }^{18}$ F-FDG PET to be more accurate than CT for initial staging, restaging, and treatment response assessment. In a brief report of 3 cases, Schuler et al. (46) presented their first experiences using combined PET/MR for high-risk sarcomas. PET/MR was used to guide neoadjuvant treatment, contributed to decision making about the appropriate time for reinitiating chemotherapy, and helped to guide biopsies of large and heterogeneous tumors.

Furthermore, DWI has been proven to be useful for the assessment of tumor cellularity in soft-tissue sarcomas and therefore may be used for monitoring the therapy response (47). In conclusion, combined PET/MR with the simultaneous acquisition of MR or fMR and PET data may have benefits for the imaging of soft-tissue sarcomas, especially for restaging and therapy response monitoring (15). 


\section{Neuroblastoma}

Neuroblastoma is the most common cancer in infancy, with a median age of $15 \mathrm{mo}(48)$. It originates from primitive neural crest cells of the sympathetic nervous system. Adrenal glands are the most common site of disease, and the presacral sympathetic chain or mediastinal sympathetic ganglia can also be affected. At the time of diagnosis, $60 \%-70 \%$ of all cases have disseminated spread, mostly involving bone marrow and bone cortex $(42,48)$.

Accurate staging of disease is essential because resection is the treatment of choice for localized tumors, whereas extensive disease requires multimodality therapy. The extent of the disease, which determines a pediatric patient's prognosis, is evaluated by ultrasound, MR, ${ }^{123}$ I-metaiodobenzylguanidine ( $\left.{ }^{123} \mathrm{I}-\mathrm{MIBG}\right)$ scintigraphy or, at present less frequently, CT $(10,48)$. Abdominal neuroblastoma typically presents as a large suprarenal mass with calcifications. MR is particularly helpful for providing information about disease extending into the spinal canal, in relation to vital structures or encased vessels, and resectability (42). ${ }^{123}$ I-MIBG scintigraphy offers high specificity and high sensitivity, $83 \%-$ $92 \%$ and $88 \%-93 \%$, respectively, particularly for bone and bone marrow diseases. MR may also demonstrate bone marrow involvement by neuroblastoma, although false-positive results have been reported (48).

The use of ${ }^{18}$ F-FDG PET for pediatric neuroblastoma is increasing, although when and in which patients it will be useful remain to be determined (49). In a comparison of ${ }^{123}$ I-MIBG scintigraphy and ${ }^{18}$ F-FDG PET for neuroblastoma, Sharp et al. (50) described an overall superiority of ${ }^{123}$ I-MIBG for the evaluation of stage 4 neuroblastoma, primarily because of better detection of bone or marrow metastases. Shulkin et al. (51) assessed the uptake of ${ }^{18} \mathrm{~F}-\mathrm{FDG}$ versus that of ${ }^{123} \mathrm{I}-\mathrm{MIBG}$ in 17 patients with neuroblastomas. They demonstrated that most neuroblastomas will concentrate ${ }^{18} \mathrm{~F}-\mathrm{FDG}$, especially before therapy, with variable uptake after therapy. Nevertheless, because ${ }^{18}$ F-FDG uptake in nontumor sites such as bone marrow or the thymus can cause potential false-positive results, ${ }^{123}$ I-MIBG has been rated superior to ${ }^{18} \mathrm{~F}-\mathrm{FDG}$ for the delineation of tumors (49-51). Thus, ${ }^{18} \mathrm{~F}-\mathrm{FDG}$ PET is regarded as beneficial for defining the distribution of neuroblastomas that fail to accumulate ${ }^{123}$ I-MIBG or show only weakly enhanced uptake, in case of any discrepancy between MR and ${ }^{123}$ I-MIBG, and when clinical symptoms or conventional imaging modalities suggest more disease than is depicted on ${ }^{123} \mathrm{I}$ MIBG scans $(50,51)$. By analogy, these settings may also be indications for combined PET/MR, which should be preferable to PET/CT in children.

Because somatostatin receptors (especially subtype 2) are expressed on the surface of some neuroblastomas, radiolabeled somatostatin analogs such as ${ }^{68} \mathrm{Ga}$ - and ${ }^{177} \mathrm{Lu}$-octreotide may be beneficial for imaging and targeted radiotherapy, especially for primary refractory or relapsed high-risk neuroblastomas (52). Given the higher sensitivity and better spatial resolution of somatostatin receptor PET than of somatostatin receptor scintigraphy for detecting somatostatin receptor-positive tumor lesions, combined PET/MR may be advantageous, especially for small liver lesions.

The use of more specific positron-emitting tracers, such as ${ }^{11} \mathrm{C}$ hydroxyephedrine (an analog of norepinephrine), ${ }^{11} \mathrm{C}$-epinephrine, ${ }^{18} \mathrm{~F}$-fluorodopamine, ${ }^{18} \mathrm{~F}$-dihydroxyphenylalanine $\left({ }^{18} \mathrm{~F}\right.$-DOPA), and $3^{\prime}$-deoxy- $3{ }^{\prime}-{ }^{18} \mathrm{~F}$-fluorothymidine, for the imaging of sympathetic nervous system tumors was studied recently and is still being evaluated $(10,49)$.

\section{Neuroendocrine Tumors}

Neuroendocrine tumors in children and adolescents are rare. They may be located in the appendix and are mostly detected incidentally through a histopathologic examination (53). Depending on the degree of tumor differentiation, PET/CT with various radiopharmaceuticals, such as ${ }^{18} \mathrm{~F}-\mathrm{FDG},{ }^{68} \mathrm{Ga}$-octreotide, ${ }^{11} \mathrm{C}$ hydroxytryptophan, and ${ }^{18} \mathrm{~F}$-DOPA, has been described as potentially improving the assessment of tumor localization and the response to therapy. For adults, MR is frequently used in conjunction with CT for the detection of neuroendocrine tumors. Rates of detection by MR have been reported to be $84 \%-95 \%$. The potential of newer MR techniques with DWI for diagnosing neuroendocrine tumors has also been described (54). Thus, combining fMR with a PET radiopharmaceutical that is highly specific for neuroendocrine tumors may improve the accuracy for diagnosing these tumors.

\section{POTENTIAL APPLICATIONS IN NEUROIMAGING}

PET and MR are the methods of choice for the imaging of neurologic neoplastic and nonneoplastic diseases. Both modalities provide complementary information about the function, metabolism, and anatomy of the brain. The outstanding role of PET imaging in detecting biochemical and molecular changes much earlier, before structural changes or clinical symptoms appear, has been described in numerous studies (55-57). fMR with DWI and MR spectroscopy has become an important noninvasive and nonradioactive tool for measuring brain activity by detecting, for instance, hemodynamic changes (in blood oxygenation and flow) induced by regional changes in neuronal activity $(1,58)$. Numerous PET tracers, mostly ${ }^{18} \mathrm{~F}$ - or ${ }^{11} \mathrm{C}$-labeled radiopharmaceuticals, are available to assess different aspects of brain function. For instance, for brain tumors, an ${ }^{18} \mathrm{~F}$-labeled amino acid such as $O$ - $\left(2-{ }^{18} \mathrm{~F}\right.$-fluoroethyl)-L-tyrosine or ${ }^{11} \mathrm{C}$-methionine has been well described (57). ${ }^{18} \mathrm{~F}-\mathrm{FDG}$ may be helpful in identifying the seizure focus in patients with refractory epilepsy or in guiding biopsies in patients with brain masses that are undefined on MR scans (55). Other promising tracers, such as the proliferation marker $3^{\prime}$-deoxy-3' ${ }^{18}$ F-fluorothymidine or ${ }^{15} \mathrm{O}$-labeled water and oxygen as perfusion markers, are currently being used in preclinical and clinical research $(55,59)$.

Until the advent of combined PET/MR, PET and MR were performed in separate examinations, mostly with secondary coregistration of MR and PET data. Especially for the analysis of brain function, combined PET/MR - with the possibility of simultaneous data acquisition-allows an improved in vivo assessment of neuropsychologic processes and a more precise description of functional, molecular, and morphologic interactive pathways. Such information may lead to an understanding of the pathophysiologic mechanisms of disorders of the central nervous system (57).

\section{Brain Tumors}

An increase in the use of PET for the evaluation of brain tumors was recently described, as was the application of fMR $(57,60)$. Thus, combined PET/MR seems to be a promising noninvasive method for the field of pediatric neuroimaging because tumors of the central nervous system are the most common solid malignancies in childhood (61). According to the literature, medulloblastoma, a tumor of primitive neuroectodermal origin, is the most common malignant brain tumor in childhood, with a peak incidence around 5-6 y of age; the next most common tumors are high-grade glioma and ependymoma (62). The histologic and bi- 
ologic heterogeneity of these malignancies requires complementary, multimodality imaging to obtain tumor kinetics and multiparametric data about tumor biology, morphology, proliferation, and vascularity $(60,63)$.

Combined PET/MR is expected to improve diagnostic accuracy and to help guide biopsy, surgery, or radiation therapy planning through the precise localization of hypermetabolic, vital tumor tissue and the accurate definition of the target volume. Furthermore, the simultaneous acquisition of PET and MR data provides precise information about tumor biology and the tumor response after antitumoral therapy (57). For brain tumors, radiolabeled amino acids such as $O$-(2- ${ }^{18} \mathrm{~F}$-fluoroethyl)-L-tyrosine, ${ }^{18} \mathrm{~F}$-DOPA, and ${ }^{11} \mathrm{C}$-methionine or the tumor proliferation marker $3^{\prime}$-deoxy$3^{\prime}{ }^{1}{ }^{18} \mathrm{~F}$-fluorothymidine have been described as sensitive and specific indicators of viable tumor and the treatment response $(57,63)$. Because the uptake of ${ }^{18}$ F-FDG by brain tumors correlates with tumor grade, the tracer can be used to image poorly differentiated, rapidly growing brain tumors such as high-grade glioma as well as cerebral lymphoma $(55,60,63)$. Because of the high level of normal ${ }^{18} \mathrm{~F}$-FDG uptake by brain tissue, there is often poor contrast of tumor lesion to background, with less accurate detection, especially of low-grade, slowly proliferating tumors. Compared with ${ }^{18} \mathrm{~F}-\mathrm{FDG},{ }^{11} \mathrm{C}$-methionine, $O-\left(2-{ }^{18} \mathrm{~F}\right.$-fluoroethyl $)$-L-tyrosine, and ${ }^{18} \mathrm{~F}-\mathrm{DOPA}$ have higher accuracies for the detection of low- and high-grade gliomas and for assessment of the treatment response because of better tumor-to-nontumor delineation $(60,63)$.

\section{Epilepsy}

Surgery has been described as superior to prolonged pharmacotherapy for medically intractable temporal lobe epilepsy (TLE), the most common partial epilepsy in adults and children. The literature describes long-term seizure control, with a seizure-free outcome in $50 \%-80 \%$ of cases, after temporal lobe resection. The success of epilepsy surgery strongly depends on the precise presurgical identification of epileptogenic foci, for which MR is the most reliable tool (64-67). Beyond electroencephalography, MR has been described as the primary imaging tool for TLE because of its high sensitivity and specificity ( $97 \%$ and $83 \%$, respectively) for hippocampal sclerosis, the most common pathologic substrate of TLE. Nevertheless, about $16 \%-55 \%$ of patients with TLE do not show any suspect lesion on MR scans (nonlesional epilepsy). Especially for this group of patients, other noninvasive functional imaging modalities - such as magnetoencephalography, ${ }^{18}$ F-FDG PET, ictal SPECT or, more recently, subtraction ictal SPECT coregistered to MR-are of great value for preoperative assessment because patients with nonlesional epilepsy have a poorer surgical outcome than those with lesions that can be detected by MR $(64-66,68)$.

The evaluation of regional cerebral perfusion in patients with epilepsy has been proven to be of significant clinical value for the identification of an epileptogenic focus. Because of the spontaneous and unpredictable nature of seizures, ictal studies are difficult to perform (67). Other noninvasive methods, such as interictal ${ }^{18} \mathrm{~F}-$ FDG PET-detecting epileptogenic foci usually by hypometabolism with a sensitivity of $70 \%-85 \%$ in patients with TLE-have been found to be useful $(5,57)$. Widjaja et al. (64) compared the application of ${ }^{18}$ F-FDG PET with that of magnetoencephalography for identifying the epileptogenic focus in 26 children who had localization-related epilepsy and no or only subtle changes on MR scans. Both modalities were found to have a complementary role in the workup of children with intractable localization-related epilepsy, improving sensitivity and specificity. This finding is con- sistent with those of other studies $(65,69)$ reporting the greatest benefit of ${ }^{18} \mathrm{~F}$-FDG PET in patients whose MR results were inconclusive or negative.

Kim et al. (67) determined the accuracy of imaging tools such as electroencephalography, MR, and ${ }^{18} \mathrm{~F}$-FDG PET in 42 pediatric patients who received epilepsy surgery (temporal lobectomies in $23 / 42$ and extratemporal resection in 19/42). Of the 23 children who had temporal lobectomies, 22 underwent an ${ }^{18} \mathrm{~F}$-FDG PET study, which determined the epileptic foci in 21 of the 22 patients and the precise localization in 16 of the 21 patients. A pathologic examination revealed hippocampus sclerosis in 16 of the 23 patients. The authors reported that the sensitivity of ${ }^{18} \mathrm{~F}-\mathrm{FDG}$ PET for the localization of temporal lesions was $72.2 \%$, approaching the sensitivity seen with MR. For extratemporal lesions, ${ }^{18} \mathrm{~F}$ FDG PET was reported to be unreliable, whereas MR seemed to be useful for both temporal and extratemporal lesions. The authors concluded that PET and MR data are complementary in establishing the correct diagnosis in TLE.

Rubí et al. (68) prospectively evaluated PET and (secondary coregistered) PET/MR data from 31 pediatric patients with nonlesional epilepsy and demonstrated that PET/MR coregistration was as accurate as PET alone for detecting hypometabolic lesions in those patients. Furthermore, the authors reported that in some cases, the initial MR diagnosis of nonlesional epilepsy changed to subtle lesional epilepsy after secondary analysis by PET/MR coregistration. They recommended coregistering PET with MR in a presurgical evaluation of all children with nonlesional epilepsy to improve epileptogenic zone identification and to delimit the anatomic boundaries of hypometabolic lesions for the purpose of guiding stereotactic neurosurgery.

Beyond localization, ${ }^{18}$ F-FDG PET plays a role in defining surgical margins because the extent of resection of the region of hypometabolism on preoperative ${ }^{18} \mathrm{~F}-\mathrm{FDG}$ PET scans is predictive of outcome after surgery for nonlesional TLE (70).

Finally, the major advantages of the application of combined PET and high-resolution MR in pediatric neuroimaging may be precise surgery planning for brain tumor biopsy or surgery and epilepsy surgery through improvements in the identification of foci of hypermetabolic, vital tumor tissue or epileptogenic foci (Figs. 2 and 3) and precise target volume delineation through topographic association of specific brain areas with tumoral or epileptogenic lesions on PET and MR scans. Furthermore, especially in younger children, the number of episodes of and the length of sedation or anesthesia may be reduced when the examinations are performed simultaneously (57).

\section{POTENTIAL APPLICATIONS IN INFECTIOUS AND INFLAMMATORY DISEASES}

Fever of unknown origin (FUO) and inflammation of unknown origin (IUO) remain challenging problems with a wide etiologic spectrum; they are predominantly caused by infections, noninfectious inflammatory diseases (such as rheumatic, autoimmune, or systemic diseases), or malignancies. There are several approaches to the diagnostic management of FUO and IUO; these include invasive and noninvasive techniques as well as imaging procedures, generally with relatively high specificity but limited sensitivity because morphologic changes may be absent in the early stages of disease (71-73). Early detection and precise localization of the focus of FUO, however, are important for guiding further diagnostic and therapeutic procedures (71). 


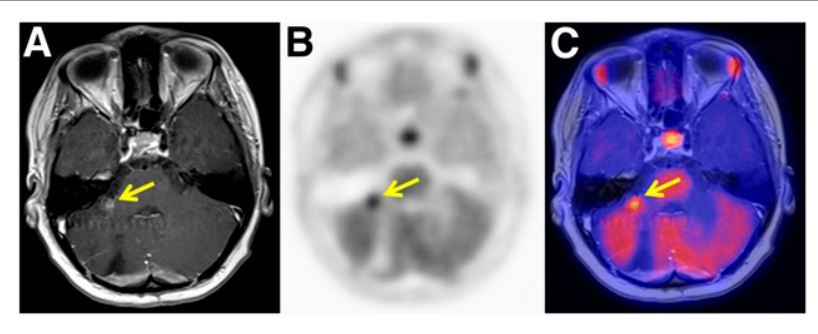

FIGURE 2. Fourteen-year-old girl with history of R0 resection and radiotherapy of glioblastoma multiforme of right cerebellum. (A) T1weighted MR imaging of brain shows contrast-enhanced suspect lesion $(9 \times 9 \mathrm{~mm})$ in right cerebellar peduncle (arrow). (B and C) Simultaneous ${ }^{11} \mathrm{C}$-methionine PET/MR imaging shows focal enhanced amino acid metabolism in this lesion, highly suggestive of tumor tissue. Histology confirmed glioblastoma relapse.

Several studies have reported that by providing information about the target organ or disease activity, ${ }^{18} \mathrm{~F}$-FDG PET/CT has a high sensitivity for the diagnosis of FUO and IUO, but there are conflicting data about its place in the diagnostic workup $(71,72$, 74). Crouzet et al. (74) reported that ${ }^{18} \mathrm{~F}-\mathrm{FDG}$ PET/CT should be considered when standard diagnostic tests, such as complete blood counts, serologic investigations, abdominal ultrasound, and chest radiography, do not provide a diagnosis in patients with FUO.

Apart from FUO, other indications for ${ }^{18} \mathrm{~F}-\mathrm{FDG}$ PET/CT in adult patients with suspected infections are spondylodiscitis, prosthetic joint infection, and vascular graft infection (73). For pediatric patients, data regarding the role of ${ }^{18} \mathrm{~F}-\mathrm{FDG}$ PET/CT in the diagnosis of infectious diseases are sparse. ${ }^{18}$ F-FDG PET/CT may provide additional information in children with pneumonia, disseminated candidiasis, cytomegalovirus spleen infection, vasculitis (e.g., in the context of Henoch-Schönlein purpura or Kawasaki disease), musculoskeletal inflammation, and inflammatory bowel disease $(73,75)$. Jasper et al. (72) investigated the diagnostic role of ${ }^{18}$ F-FDG PET and PET/CT in children with unexplained inflammatory signs. In children with a final diagnosis $(n=37$; $54 \%$ ), PET was considered diagnostically helpful in $73 \%$. For inflammatory bowel disease in children and adolescents, Berthold et al. (75) discussed ${ }^{18}$ F-FDG PET as a valuable tool for determining the extent and degree of inflammation, especially in parts of the small bowel that are inaccessible to endoscopy. On the basis of the results of Ream et al. (76) for pediatric small-bowel Crohn disease, DWI could be used to improve the detection of affected

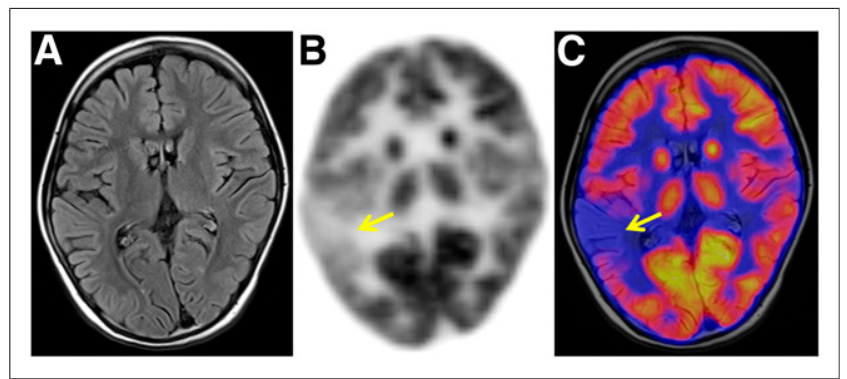

FIGURE 3. Seven-year-old girl with 2-y history of medically intractable focal epileptic seizures suspected of originating in right hemisphere. (A) T2-weighted MR imaging of brain shows no clear epileptogenic lesion. (B and C) Interictal simultaneous ${ }^{18}$ F-FDG PET/MR imaging shows hypometabolism in right temporal lobe (arrows), suggestive of epileptogenic focus. small-bowel segments. Obviously, apparent diffusion coefficient values may also be helpful for assessing the degree of inflammation in Crohn disease because distinguishing fibrotic and inflammatory bowel strictures is usually difficult. Thus, the approach of combined PET/MR with various MR techniques may be beneficial for the accurate detection and monitoring of disease activity in inflammatory bowel disease, especially when complementary imaging is needed to characterize intestinal lesions $(1,76)$.

A limitation of ${ }^{18}$ F-FDG may be the physiologic distribution in several tissues, such as the brain, the genitourinary tract, the myocardium (with variably high activity), and the bowel, making the diagnosis of inflammation in these sites challenging. Thus, another approach is to use different, more specific radiopharmaceuticals for inflammation localization. Especially for intestinal inflammation, white blood cells labeled with ${ }^{18} \mathrm{~F}-\mathrm{FDG}$ have been described as a feasible alternative with a high negative predictive value for the quantitative assessment of bowel inflammation (77).

For the detection of neuroinflammation, several new PET tracers, such as radiolabeled cyclooxygenase 2 inhibitors, are currently under investigation.

Glaudemans et al. (1) described a significant potential of hybrid whole-body PET/MR for diagnosing infectious and inflammatory diseases by significantly improving the sensitivity and specificity of the diagnosis and follow-up treatment. The authors expected hybrid whole-body PET/MR to provide a more accurate assessment of the extent and exact localization of inflammatory lesions than PET/CT or PET alone. The main clinical impact was expected in soft-tissue lesions that are especially prone to motion artifacts, such as vascular and cardiac infections and inflammatory bowel disease. Additionally, fMR promises to provide a deeper insight into the special characteristics and dynamics of the inflammatory process.

\section{OTHER POTENTIAL APPLICATIONS}

\section{Pediatric Cardiology}

Beside transthoracic echocardiography, cardiac MR is an established noninvasive modality in pediatric cardiology; it is used for visualization of the coronary arterial system and assessment of coronary arterial blood flow, for example, in children with congenitally malformed hearts or after switch operations (78). The main impact of cardiac PET is the quantification of myocardial blood flow and coronary flow reserve to improve the detection of preclinical and multivessel coronary atherosclerosis (79). Because this diagnosis is rarely seen in pediatric patients, cardiac PET plays only a minor role in these patients.

\section{Maternal-Fetal Medicine}

Jones and Budinger (80) recently described a potential research application of PET/MR imaging with small amounts of short-lived radiolabeled biomarkers - the study of the human fetus in vivo in the field of modern preventive medicine. We believe that there is a need to investigate various aspects of fetal-maternal function in vivo, including the measurement of oxygen uptake in the fetal brain, nutrient delivery, metabolism, or placenta transport, to improve understanding of the influence of these parameters on lifelong health.

\section{CONCLUSION}

Combined PET/MR will provide new perspectives in noninvasive imaging to improve understanding of the dynamics and 
characteristics of tumoral or nontumoral processes. Combined $\mathrm{PET} / \mathrm{MR}$ is expected to have an emerging role in pediatric diagnostic imaging because the lower radiation burden of this modality than of PET/CT is an important advantage. Compared with separately acquired, coregistered PET and MR imaging studies, simultaneous PET/MR as a 1-step diagnostic imaging workup not only will improve image fusion and image resolution but also may enhance patient convenience; reductions in total scan time, repeated examinations, and repeated sedation or anesthesia can reduce patient anxiety. However, the full potential and the added value of simultaneous PET/MR are not yet evident, and further investigation is needed.

In oncology patients, the simultaneous acquisition of data from fMR with, for example, DWI and PET may be of great value, especially for restaging purposes and therapy response monitoring. For the staging of brain tumors, PET/MR allows more precise target volume delineation and therefore also more accurate planning of biopsy or surgery, with a resultant marked impact on patients' outcomes. The approach of combined PET/MR with various MR techniques may also be beneficial for determining the exact localization and extent of inflammatory processes, especially when complementary imaging is needed to characterize suspect lesions. Nevertheless, the added diagnostic value of fMR sequences still must be demonstrated before implementation into PET/ MR protocols, especially for pediatric patients, for whom imaging protocols should be kept as simple as possible to maintain their tolerance.

\section{DISCLOSURE}

No potential conflict of interest relevant to this article was reported.

\section{REFERENCES}

1. Glaudemans AW, Quintero AM, Signore A. PET/MRI in infectious and inflammatory diseases: will it be a useful improvement? Eur J Nucl Med Mol Imaging. 2012;39:745-749.

2. Pichler BJ, Kolb A, Nägele T, Schlemmer HP. PET/MRI: paving the way for the next generation of clinical multimodality imaging applications. J Nucl Med. 2010;51:333-336.

3. Pichler BJ, Wehrl HF, Kolb A, Judenhofer MS. Positron emission tomography/ magnetic resonance imaging: the next generation of multimodality imaging? Semin Nucl Med. 2008;38:199-208.

4. Bailey DL, Barthel H, Beyer T, et al. Summary Report of the First International Workshop on PET/MR Imaging, March 19-23, 2012, Tübingen, Germany. Mol Imaging Biol. 2013;15:361-371.

5. Drzezga A, Souvatzoglou M, Eiber M, et al. First clinical experience with integrated whole-body PET/MR: comparison to PET/CT in patients with oncologic diagnoses. J Nucl Med. 2012;53:845-855.

6. Wiesmüller M, Quick HH, Navalpakkam B, et al. Comparison of lesion detection and quantitation of tracer uptake between PET from a simultaneously acquiring whole-body PET/MR hybrid scanner and PET from PET/CT. Eur J Nucl Med Mol Imaging. 2013;40:12-21.

7. Schwenzer NF, Pfannenberg C, Reischl G, Werner MK, Schmidt H. Application of MR/PET in oncologic imaging [in German]. Rofo. 2012;184:780-787.

8. Hirsch FW, Sattler B, Sorge I, et al. PET/MR in children: initial clinical experience in paediatric oncology using an integrated PET/MR scanner. Pediatr Radiol. 2013;43:860-875.

9. Jadvar H, Colletti PM. Competitive advantage of PET/MRI. Eur J Radiol. 2014;83:84-94.

10. Jadvar H, Connolly LP, Fahey FH, Shulkin BL. PET and PET/CT in pediatric oncology. Semin Nucl Med. 2007;37:316-331.

11. Punwani S, Taylor SA, Saad ZZ, et al. Diffusion-weighted MRI of lymphoma: prognostic utility and implications for PET/MRI? Eur J Nucl Med Mol Imaging. 2013;40:373-385.
12. Darge K, Jaramillo D, Siegel MJ. Whole-body MRI in children: current status and future applications. Eur J Radiol. 2008;68:289-298.

13. Yankeelov TE, Peterson TE, Abramson RG, et al. Simultaneous PET-MRI in oncology: a solution looking for a problem? Magn Reson Imaging. 2012;30: 1342-1356.

14. Goo HW. Regional and whole-body imaging in pediatric oncology. Pediatr Radiol. 2011;41(suppl):S186-S194.

15. Buchbender C, Heusner TA, Lauenstein TC, Bockisch A, Antoch G. Oncologic PET/MRI, part 2: bone tumors, soft-tissue tumors, melanoma, and lymphoma. J Nucl Med. 2012;53:1244-1252.

16. Buchbender C, Heusner TA, Lauenstein TC, Bockisch A, Antoch G. Oncologic PET/MRI, part 1: tumors of the brain, head and neck, chest, abdomen, and pelvis. J Nucl Med. 2012;53:928-938.

17. Montravers F, McNamara D, Landman-Parker J, et al. $\left[{ }^{18} \mathrm{~F}\right] \mathrm{FDG}$ in childhood lymphoma: clinical utility and impact on management. Eur J Nucl Med Mol Imaging. 2002;29:1155-1165.

18. Kluge R, Kurch L, Montravers F, Mauz-Körholz C. FDG PET/CT in children and adolescents with lymphoma. Pediatr Radiol. 2013;43:406-417.

19. Gu J, Chan T, Zhang J, Leung AY, Kwong YL, Khong PL. Whole-body diffusionweighted imaging: the added value to whole-body MRI at initial diagnosis of lymphoma. AJR Am J Roentgenol. 2011;197:W384-W391.

20. Kluge R, Körholz D. Role of FDG-PET in staging and therapy of children with Hodgkin lymphoma. Klin Padiatr. 2011;223:315-319.

21. London K, Cross S, Onikul E, Dalla-Pozza L, Howman-Giles R. ${ }^{18}$ F-FDG PET/ $\mathrm{CT}$ in paediatric lymphoma: comparison with conventional imaging. Eur J Nucl Med Mol Imaging. 2011;38:274-284.

22. Krohmer S, Sorge I, Krausse A, et al. Whole-body MRI for primary evaluation of malignant disease in children. Eur J Radiol. 2010;74:256-261.

23. Lin C, Luciani A, Itti E, et al. Whole-body diffusion magnetic resonance imaging in the assessment of lymphoma. Cancer Imaging. 2012;12:403-408.

24. Purz S, Mauz-Körholz C, Körholz D, et al. $\left[{ }^{18} \mathrm{~F}\right]$ fluorodeoxyglucose positron emission tomography for detection of bone marrow involvement in children and adolescents with Hodgkin's lymphoma. J Clin Oncol. 2011;29:35233528

25. Wu LM, Chen FY, Jiang XX, Gu HY, Yin Y, Xu JR. ${ }^{18}$ F-FDG PET, combined FDG-PET/CT and MRI for evaluation of bone marrow infiltration in staging of lymphoma: a systematic review and meta-analysis. Eur J Radiol. 2012;81:303-311.

26. Pfluger T, Melzer HI, Mueller WP, et al. Diagnostic value of combined ${ }^{18} \mathrm{~F}$-FDG PET/MRI for staging and restaging in paediatric oncology. Eur J Nucl Med Mol Imaging. 2012;39:1745-1755.

27. Biederer J, Beer M, Hirsch W, et al. MRI of the lung $(2 / 3)$. Why ... when ... how? Insights Imaging. 2012;3:355-371.

28. Hirsch W, Sorge I, Krohmer S, Weber D, Meier K, Till H. MRI of the lungs in children. Eur J Radiol. 2008;68:278-288.

29. Juweid ME, Stroobants S, Hoekstra OS, et al. Use of positron emission tomography for response assessment of lymphoma: consensus of the Imaging Subcommittee of International Harmonization Project in Lymphoma. J Clin Oncol. 2007;25:571-578.

30. Hutchings M, Loft A, Hansen M, et al. FDG-PET after two cycles of chemotherapy predicts treatment failure and progression-free survival in Hodgkin lymphoma. Blood. 2006;107:52-59.

31. Furth C, Steffen IG, Amthauer H, et al. Early and late therapy response assessment with $\left[{ }^{18} \mathrm{~F}\right]$ fluorodeoxyglucose positron emission tomography in pediatric Hodgkin's lymphoma: analysis of a prospective multicenter trial. J Clin Oncol. 2009;27:4385-4391.

32. Bhatia S. Long-term complications of therapeutic exposures in childhood: lessons learned from childhood cancer survivors. Pediatrics. 2012;130: 1141-1143.

33. Chen Y, Zhong J, Wu H, Chen N. The clinical application of whole-body diffusion-weighted imaging in the early assessment of chemotherapeutic effects in lymphoma: the initial experience. Magn Reson Imaging. 2012;30:165-170.

34. Kwee TC, Takahara T, Vermoolen MA, et al. Whole-body diffusion-weighted imaging for staging malignant lymphoma in children. Pediatr Radiol. 2010;40: 1592-1602.

35. ESMO/European Sarcoma Network Working Group. Bone sarcomas: ESMO Clinical Practice Guidelines for diagnosis, treatment and follow-up. Ann Oncol. 2012;23(suppl 7):vii100-vii109.

36. Denecke T, Hundsdörfer P, Misch D, et al. Assessment of histological response of paediatric bone sarcomas using FDG PET in comparison to morphological volume measurement and standardized MRI parameters. Eur J Nucl Med Mol Imaging. 2010;37:1842-1853.

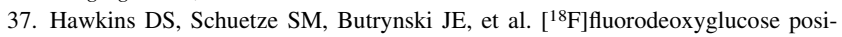
tron emission tomography predicts outcome for Ewing sarcoma family of tumors. J Clin Oncol. 2005;23:8828-8834. 
38. London K, Stege C, Cross S, et al. ${ }^{18}$ F-FDG PET/CT compared to conventional imaging modalities in pediatric primary bone tumors. Pediatr Radiol. 2012;42: 418-430.

39. Uhl M, Saueressig U, van Buiren M, et al. Osteosarcoma: preliminary results of in vivo assessment of tumor necrosis after chemotherapy with diffusion- and perfusion-weighted magnetic resonance imaging. Invest Radiol. 2006;41: $618-623$.

40. Völker T, Denecke T, Steffen I, et al. Positron emission tomography for staging of pediatric sarcoma patients: results of a prospective multicenter trial. J Clin Oncol. 2007;25:5435-5441.

41. Hawkins DS, Spunt SL, Skapek SX; COG Soft Tissue Sarcoma Committee. Children's Oncology Group's 2013 blueprint for research: soft tissue sarcomas. Pediatr Blood Cancer. 2013;60:1001-1008.

42. Servaes S, Epelman M, Pollock A, Shekdar K. Pediatric malignancies: synopsis of current imaging techniques. Cancer Treat Res. 2008;143:469-491.

43. Ricard F, Cimarelli S, Deshayes E, Mognetti T, Thiesse P, Giammarile F. Additional benefit of F-18 FDG PET/CT in the staging and follow-up of pediatric rhabdomyosarcoma. Clin Nucl Med. 2011;36:672-677.

44. Evilevitch V, Weber WA, Tap WD, et al. Reduction of glucose metabolic activity is more accurate than change in size at predicting histopathologic response to neoadjuvant therapy in high-grade soft-tissue sarcomas. Clin Cancer Res. 2008;14:715-720.

45. Piperkova E, Mikhaeil M, Mousavi A, et al. Impact of PET and CT in PET/CT studies for staging and evaluating treatment response in bone and soft tissue sarcomas. Clin Nucl Med. 2009;34:146-150.

46. Schuler MK, Richter S, Beuthien-Baumann B, et al. PET/MRI imaging in highrisk sarcoma: first findings and solving clinical problems. Case Rep Oncol Med. 2013;2013:793927.

47. Schnapauff D, Zeile M, Niederhagen MB, et al. Diffusion-weighted echo-planar magnetic resonance imaging for the assessment of tumor cellularity in patients with soft-tissue sarcomas. J Magn Reson Imaging. 2009;29:1355-1359.

48. Sharp SE, Parisi MT, Gelfand MJ, Yanik GA, Shulkin BL. Functional-metabolic imaging of neuroblastoma. Q J Nucl Med Mol Imaging. 2013;57:6-20.

49. Mueller WP, Coppenrath E, Pfluger T. Nuclear medicine and multimodality imaging of pediatric neuroblastoma. Pediatr Radiol. 2013;43:418-427.

50. Sharp SE, Shulkin BL, Gelfand MJ, Salisbury S, Furman WL. ${ }^{123}$ I-MIBG scintigraphy and ${ }^{18}$ F-FDG PET in neuroblastoma. J Nucl Med. 2009;50:1237-1243.

51. Shulkin BL, Hutchinson RJ, Castle VP, Yanik GA, Shapiro B, Sisson JC. Neuroblastoma: positron emission tomography with 2-[fluorine-18]-fluoro-2-deoxyD-glucose compared with metaiodobenzylguanidine scintigraphy. Radiology. 1996;199:743-750

52. Gains JE, Bomanji JB, Fersht NL, et al. ${ }^{177}$ Lu-DOTATATE molecular radiotherapy for childhood neuroblastoma. J Nucl Med. 2011;52:1041-1047.

53. Boxberger N, Redlich A, Böger C, et al. Neuroendocrine tumors of the appendix in children and adolescents. Pediatr Blood Cancer. 2013;60:65-70.

54. Leung D, Schwartz L. Imaging of neuroendocrine tumors. Semin Oncol. 2013;40:109-119.

55. Singhal T. Positron emission tomography applications in clinical neurology. Semin Neurol. 2012;32:421-431.

56. Garibotto V, Heinzer S, Vulliemoz S, et al. Clinical applications of hybrid PET/ MRI in neuroimaging. Clin Nucl Med. 2013;38:e13-e18.

57. Catana C, Drzezga A, Heiss WD, Rosen BR. PET/MRI for neurologic applications. J Nucl Med. 2012;53:1916-1925.

58. Freilich ER, Gaillard WD. Utility of functional MRI in pediatric neurology. Curr Neurol Neurosci Rep. 2010;10:40-46.

59. Schwaiger M, Wester HJ. How many PET tracers do we need? J Nucl Med. 2011;52(suppl):36S-41S
60. Kim S, Salamon N, Jackson HA, Blüml S, Panigrahy A. PET imaging in pediatric neuroradiology: current and future applications. Pediatr Radiol. 2010; 40:82-96.

61. Hipp SJ, Steffen-Smith EA, Patronas N, et al. Molecular imaging of pediatric brain tumors: comparison of tumor metabolism using ${ }^{18}$ F-FDG-PET and MRSI. $J$ Neurooncol. 2012;109:521-527.

62. Gajjar A, Packer RJ, Foreman NK, et al. Children's Oncology Group's 2013 blueprint for research: central nervous system tumors. Pediatr Blood Cancer. 2013;60:1022-1026.

63. Warren KE. Noninvasive assessment of pediatric brain tumors. Cancer Biol Ther. 2009;8:1881-1888.

64. Widjaja E, Shammas A, Vali R, et al. FDG-PET and magnetoencephalography in presurgical workup of children with localization-related nonlesional epilepsy. Epilepsia. 2013;54:691-699.

65. Gok B, Jallo G, Hayeri R, Wahl R, Aygun N. The evaluation of FDG-PET imaging for epileptogenic focus localization in patients with MRI positive and MRI negative temporal lobe epilepsy. Neuroradiology. 2013;55:541-550.

66. Seo JH, Holland K, Rose D, et al. Multimodality imaging in the surgical treatment of children with nonlesional epilepsy. Neurology. 2011;76:41-48.

67. Kim JT, Bai SJ, Choi KO, et al. Comparison of various imaging modalities in localization of epileptogenic lesion using epilepsy surgery outcome in pediatric patients. Seizure. 2009;18:504-510.

68. Rubí S, Setoain X, Donaire A, et al. Validation of FDG-PET/MRI coregistration in nonlesional refractory childhood epilepsy. Epilepsia. 2011;52:2216-2224.

69. Won HJ, Chang KH, Cheon JE, et al. Comparison of MR imaging with PET and ictal SPECT in 118 patients with intractable epilepsy. AJNR Am J Neuroradiol. 1999;20:593-599.

70. Vinton AB, Carne R, Hicks RJ, et al. The extent of resection of FDG-PET hypometabolism relates to outcome of temporal lobectomy. Brain. 2007;130: $548-560$.

71. Balink H, Collins J, Bruyn GA, Gemmel F. F-18 FDG PET/CT in the diagnosis of fever of unknown origin. Clin Nucl Med. 2009;34:862-868.

72. Jasper N, Däbritz J, Frosch M, Loeffler M, Weckesser M, Foell D. Diagnostic value of $\left[{ }^{18} \mathrm{~F}\right]-\mathrm{FDG}$ PET/CT in children with fever of unknown origin or unexplained signs of inflammation. Eur J Nucl Med Mol Imaging. 2010;37:136-145.

73. del Rosal T, Goycochea WA, Méndez-Echevarría A, et al. ${ }^{18}$ F-FDG PET/CT in the diagnosis of occult bacterial infections in children. Eur J Pediatr. 2013;172: 1111-1115.

74. Crouzet J, Boudousq V, Lechiche C, et al. Place of ${ }^{18}$ F-FDG-PET with computed tomography in the diagnostic algorithm of patients with fever of unknown origin. Eur J Clin Microbiol Infect Dis. 2012;31:1727-1733.

75. Berthold LD, Steiner D, Scholz D, Alzen G, Zimmer KP. Imaging of chronic inflammatory bowel disease with ${ }^{18}$ F-FDG PET in children and adolescents. Klin Padiatr. 2013;225:212-217.

76. Ream JM, Dillman JR, Adler J, et al. MRI diffusion-weighted imaging (DWI) in pediatric small bowel Crohn disease: correlation with MRI findings of active bowel wall inflammation. Pediatr Radiol. 2013;43:1077-1085.

77. Dumarey N, Egrise D, Blocklet D, et al. Imaging infection with ${ }^{18} \mathrm{~F}-\mathrm{FDG}$-labeled leukocyte PET/CT: initial experience in 21 patients. J Nucl Med. 2006;47:625632.

78. Friedman AH, Fogel MA, Stephens P Jr, et al. Identification, imaging, functional assessment and management of congenital coronary arterial abnormalities in children. Cardiol Young. 2007;17:56-67.

79. Di Carli MF, Dorbala S. Cardiac PET-CT. J Thorac Imaging. 2007;22:101-106.

80. Jones T, Budinger TF. The potential for low-dose functional studies in maternalfetal medicine using PET/MR imaging. J Nucl Med. 2013;54:2016-2017. 